\title{
Molecular diagnosis and therapy of hepatocellular carcinoma: achievements and challenges
}

\author{
Xiao-Dong Zhang, Man Zhao \\ Department of Cancer Research, College of Life Sciences, Nankai University, Tianjin 300071, China.
}

Correspondence to: Prof. Xiao-Dong Zhang, Department of Cancer Research, College of Life Sciences, Nankai University, 94 Weijin Road, Tianjin 300071, China. E-mail: zhangxd@nankai.edu.cn

How to cite this article: Zhang XD, Zhao M. Molecular diagnosis and therapy of hepatocellular carcinoma: achievements and challenges. Hepatoma Res 2019;5:14. http://dx.doi.org/10.20517/2394-5079.2018.117

Received: 12 Dec 2018 First Decision: 18 Mar 2019 Revised: 8 Apr 2019 Accepted: 26 Apr 2019 Published: 10 May 2019

Science Editor: Guang-Wen Cao Copy Editor: Cai-Hong Wang Production Editor: Huan-Liang Wu

\begin{abstract}
Hepatocellular carcinoma (HCC) is often associated with pre-existing chronic liver pathologies of different origin infections of hepatitis B virus (HBV) and hepatitis C virus. Clinically, the diagnosis and therapy for HCC are very important for the prognosis of patients. However, current methods for HCC diagnosis and therapy have no an optimal accuracy due to the tumor heterogeneity and the frequent late diagnosis. This review summarizes the new advances in molecular diagnosis and therapy of HCC, based on the recent novel biomarkers and new therapeutic strategies for HCC, including alpha-fetoprotein-L3, glypican-3, heat shock protein 90, dickkopf WNT signaling pathway inhibitor 1, paraoxonase 1, highly up-regulated in liver cancer. Moreover, epigenetic regulation, signal pathway, cellular and molecular targets for the immunotherapy, tumor microenviroment and genome sequencing analysis may serve as the molecular expression signatures in clinical practice. For promising new treatment strategy of HCC, targeting molecular therapy based on the restoration of tumor suppressor genes lost and inhibition of oncogenic genes is attractive. The new clinical trials for other molecular-targeted agents, including pembrolizumab, nivolumab, tivantinib, lenvatinib, cabozantinib, and ramucirumab, are ongoing in clinic. Interestingly, anti-HBV drugs display an amazing therapy for HBV-related HCC. In future, the global determination of more biomarkers may provide new insights into the diagnosis of HCC. More importantly, the diagnostic markers should be used to trace patient's follow-up disease progression, guiding doctors to judge and prescribe drugs for status of an illness, prognosis and other processes.
\end{abstract}

Keywords: Molecular diagnosis, therapy, hepatocellular carcinoma, hepatitis B virus, hepatitis $C$ virus

\section{INTRODUCTION}

Hepatocellular carcinoma (HCC) is a serious health issue globally. The increased trends of HCC will remain until $2030^{[1]}$. According to the World Health Organization, HCC is the fifth most common cancer

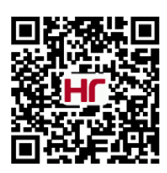


worldwide and the second most common cause of cancer-related death in 2015. Hepatitis B virus (HBV) or hepatitis C virus (HCV) infection accounting for $80 \%-90 \%$ of all HCC cases are well-known major risk factors for the development of HCC. The other risk factors, such as aflatoxin B1 exposure, alcoholic and non-alcoholic liver cirrhosis, obesity, diabetes, vitamin D deficiency, are involved in HCC occurrence ${ }^{[2,3]}$. Although treatment with HBV and HCV infection by some recent antiviral therapies is available, virally mediated hepatocarcinogenesis is still the etiology for the majority of HCC cases worldwide ${ }^{[4]}$. In patients with advanced HCC, there is low response to chemotherapy, and sorafenib is the only standard treatment recommended in international guidelines ${ }^{[5]}$. Thus, it is very important to identify novel diagnosis biomarkers and therapeutic targets for prognosis of HCC.

Molecular mechanisms of malignant cells will lead to the development of successful HCC therapies. NcRNAs and epigenetic regulation have been considered as a potential non-invasive biomarkers due to their experimental and clinical versatility. Whole-genome sequencing analysis has promoted molecular profiles transduced in expression "signatures" which will help in the comprehension of liver physiopathology. HCC etiology seems to be a factor that should be included in several clinic association studies.

The development of novel and useful biomarkers can be employed as a screening strategy for early diagnosis and prognosis in these high-risk populations, since HCC presents a high mortality rate ${ }^{[6]}$. Because late diagnosis, resistance to treatment, tumor recurrence, and metastasis cause to low survival, it is essential for developing novel diagnostics and therapeutics of $\mathrm{HCC}^{[7]}$. However, current methods for HCC diagnosis and therapy have no an optimal accuracy due to the tumor heterogeneity and the frequent late diagnosis. Therefore, the most urgent needs for early diagnosis and novel therapies of HCC should be developed.

\section{DIAGNOSIS FOR HCC}

The present methods for diagnosis of HCC can be divided into the following major aspects: magnetic resonance imaging, abdominal ultrasonography, and contrast-enhanced computed tomography, liver biopsy, and serological test. However, the diagnostic effectiveness of above technologies is not very satisfied, particularly for the diagnosis of small lesions and early diagnosis of HCC. The abdominal ultrasound is an operator-dependent test. Liver biopsy is an invasive method not exempt of mortality risk ${ }^{[8]}$. Therefore, the serological test should be developed. In this review, we focused on the advances of gene diagnosis for HCC.

\section{MiRNAs serve as HCC diagnostic markers}

As we know, cellular miRNAs released into the extracellular circulation system can be detected by serological test. Owing to circulating miRNAs relevant to HCC, miRNAs may serve as potential biomarkers. Thus, some circulating miRNAs can be considered as representative of certain pathological conditions. Moreover, circulating miRNAs possess accessibility well and high stability in the detection system, particularly for supervision of early stage, pre-symptomatic diseases in at-risk patients ${ }^{[9]}$. It has been reported that a serum diagnostic test, based on a 34-miRNAs signature, can recognize the early stage lung cancer with $80 \%$ accuracy $^{[10]}$.

MiRNAs may be used as biomarkers for prognosis or diagnosis in HCC. Down-regulation of miR-let-7g, miR-22, miR-26, miR-29, miR-99a, miR-124, miR-139, miR-145 and miR-199b is involved in the cell's life activities, including proliferation, apoptosis, angiogenesis, disease recurrence, disease-free survival (DFS) and poor prognosis ${ }^{[1-19]}$. On the contrary, the increase of miR-10b, miR-17-5p, miR-21, miR-135a, miR-155, miR-182, miR-221 and miR-222 is taken part in the metastasis, angiogenesis and poor prognosis ${ }^{[20-26]}$. Additionally, miRNA profiling categorizes HCC into three main parts ${ }^{[27]}$. The above discoveries display the important value of miRNA detection in prediction of HCC survival. Some microRNAs may be used for HCC diagnosis. MiR-101 in serum sample was $95.5 \%$ for sensitivity and $90.2 \%$ for specificity, respectively ${ }^{[28]}$. MiR-18a in serum sample was $86.1 \%$ for sensitivity and $75 \%$ for specificity, respectively ${ }^{[29]}$. The expression of miR-25 was 
Table 1. Serum diagnostic markers in hepatocellular carcinoma

\begin{tabular}{|c|c|c|}
\hline Biomarkers & Sensitivity (\%) & Specificity (\%) \\
\hline $\mathrm{AFP}^{[6]}$ & 60.0 & 85.0 \\
\hline AFP-L $3^{[6]}$ & 84.9 & 86.4 \\
\hline$D C P^{[6]}$ & 80.0 & 81.0 \\
\hline$A F P+A F P-L 3+D C P^{[6]}$ & 94.3 & 86.4 \\
\hline $\mathrm{PON}^{[6]}$ & 41.6 & 85.7 \\
\hline Fuc-PON1 $1^{[6]}$ & 79.1 & 53.5 \\
\hline Hsp90 $\alpha^{[53]}$ & 93.32 & 90.27 \\
\hline Hsp90 $\alpha+$ AFP $^{[53]}$ & 93.70 & 94.40 \\
\hline $\mathrm{MiR}-101^{[28]}$ & 95.5 & 90.2 \\
\hline MiR-18a $a^{[29]}$ & 86.1 & 75 \\
\hline
\end{tabular}

AFP: alpha-fetoprotein; AFP-L3: lens culinaris agglutinin (LCA)-reactive AFP; DCP: C-carboxy prothrombin; PON1: paraoxonase 1; FucPON1: PON1-fucosylated level protein; HSP90 $\alpha$ : heat shock protein 90 alpha

significantly up-regulated in HCC tissues, which may be used in HCC prognosis ${ }^{[30]}$. And miR-155 reflected tumor recurrence, micro-vascular invasion and recurrence-free survival ${ }^{[31]}$ [Table 1].

Furthermore, it has been reported that miR-500 is highly expressed in the sera of HCC patients. While, after surgical treatment, the expression level is reduced ${ }^{[32]}$. What's more, other miRNAs including miR-25, miR-375 and let-7f, can also be used for distinguishing HCC from normal tissue ${ }^{[33]}$. Thus, the levels of extracellular miRNA expression are steady in the body circulation. It suggests that miRNAs may be used as biomarkers for HCC diagnosis.

\section{LncRNAs function as HCC diagnostic markers}

Long non-coding RNAs (lncRNAs) are a subgroup of non-coding RNA transcripts greater than 200 nucleotides in length with little or no protein-coding potential. Emerging evidence indicates that lncRNAs may play important regulatory roles in the pathogenesis and progression of human cancers, including HCC. Certain lncRNAs may be used as diagnostic or prognostic markers for HCC, a serious malignancy with increasing morbidity and high mortality rates worldwide. LncRNA HOX transcript antisense intergenic RNA (Hotair) which can bind to lysine-specific demethylase 1 (LSD1) is a 2.2 kilobase ncRNA residing in the HOXC locus. Hotair serves as a scaffold of histone modification complexes including LSD1 and polycombrepressive complex 2, leading to the development of various tumors ${ }^{[34,35]}$. For example, owing to Hotair serving as a scaffold, we found that HBXIP/Hotair/LSD1 complex function as a critical effector of c-Myc in transcriptional activation of downstream target genes ${ }^{[36]}$. Silencing Hotair increased response of HepG2 to apoptosis stimulation from TNF- $\alpha$ and chemo-drug Cisplatin and Doxorubicin on a dose-manner ${ }^{[37]}$. Highly upregulated in liver cancer (HULC) was detected in 63\% (19/30) of the HCC patient's serum, which was much higher than in the healthy control group $(10 \%, 2 / 20)^{[38]}$. Among the HCC patients, HULC detection frequencies increase with Edmondson grades. The detection rates are $14 \%, 62 \%$, and $100 \%$ for Edmondson grades I-II, II-III, and III-IV, respectively ${ }^{[38]}$. HULC was detected more frequently in the plasma of $\mathrm{HBV}^{+}$ HCC patients $(90 \%)$ than in HBV-HCC patients $(25 \%)^{[38]}$. These observations indicate that the presence of HULC is an indication of HCC and its progression. Interestingly, HULC contributes to the abnormal lipid metabolism in HCC cells ${ }^{[39]}$. Hepatitis B virus X protein ( $\mathrm{HBx}$ ) is able to raise the expression of HULC in both normal liver L-O2 cells and liver cancer HepG2 cells ${ }^{[40]}$. In addition, HULC significantly enhances the hepatocellular proliferation by promoting the HMGA2 expression by sequestration of the microRNA-186 in HCC $^{[41]}$. Therefore, the data support the clinical usage of HULC IncRNA as a potential biomarker for HCC diagnosis and prognosis.

Taken together, the development of lncRNA expression profiling using high-throughput technology for specific HCC biomarkers will no doubt lead to more accurate and precise clinical decision-making having the consequence of better patient care in the future. While the first miRNA (lin-4) was identified two 
decades ago, other ncRNAs including lncRNAs, snoRNAs, siRNAs and piRNAs have been surfaced and proved to be essential players in cancer pathogenesis ${ }^{[42]}$. Therefore, the combination of IncRNAs with other ncRNAs should not be underestimated in the onset and progression of HCC.

\section{Epigenetic markers}

DNA methylation affects the phenotype mainly through expression of the corresponding genes, methylation profiles could reflect the biological characteristics of HCC if "passive methylation" could be eliminated appropriately. A number of results have shown the predictive value of selected methylation events on survival ${ }^{[43]}$. After hepatectomy, the methylation map of liver may reflect the recurrence-free survival of HCC patients ${ }^{[44]}$. It has been reported that the determination of DNA methylation as a potential tumor marker is able to monitor the circulating tumor DNA in plasma samples ${ }^{[45]}$. High levels of trimethylated histone $\mathrm{H} 3$ lysine 4 (H3K4me3) were usually accompanied by the decreased overall survival and poor prognosis in $\mathrm{HCC}^{[46]}$. Another research also indicated that high levels of $\mathrm{H} 3 \mathrm{~K} 27 \mathrm{me} 3$ forecasted poor prognosis and aggressive tumor characteristics, such as large tumor size, vascular invasion, multiplicity of tumors and poor differentiation ${ }^{[47]}$. To better understand the roles in HCC, more studies using accurate detection methods, such as ChIP-sequencing, may be developed to evaluate these specific DNA-protein modifications.

About the traditional biomarkers, because the false negative rate of alpha-fetoprotein (AFP) is about $40 \%$ for early-stage HCC patients, 15\%-30\% of all the patients, even patients with advanced HCC, AFP levels remain normal $^{[48]}$. Lens culinaris agglutinin (LCA)-reactive AFP (AFP-L3) as an isoform of AFP may improve the detective rate for small lesion of liver cancer ${ }^{[49]}$. It has been reported that C-carboxy prothrombin (DCP) has a higher specificity for HCC than AFP but is less sensitive. Thus, the combination of AFP, AFP-L3, and DCP seems to significantly improve HCC diagnostic accuracy ${ }^{[50]}$. The combination of Glypican-3 (GPC3) as a novel tumor marker of HCC with AFP proves the sensitivity but not the specificity in HCC diagnosis ${ }^{[51]}$. Moreover, the combination of the NH2-terminal portion of GPC3 which is also called soluble GPC3 with AFP can improve overall sensitivity from $50 \%$ to $72 \%{ }^{[52]}$. Interestingly, the dynamic changes of plasma Hsp90 $\alpha$ in liver cancer patients can detect the condition of treatment, such as surgery and interventional therapy ${ }^{\left[{ }^{33]}\right.}$. Serum Dickkopf WNT signaling pathway inhibitor 1 (DKK1) was reported to be a useful biomarker for diagnosis of HCC by a large-scale and multicenter study ${ }^{[54]}$. Paraoxonase 1 (PON1) has been proposed as a circulating protein biomarker since high serum levels in HCC patients concomitantly infected with HCV infection has been observed ${ }^{[55]}$. PON1-fucosylated level protein has been helpful in distinguishing early HCC from liver cirrhosis patients even with low AFP levels ${ }^{[56]}$.

\section{THERAPY FOR HCC}

\section{Prevention and management of HBV infection}

Based on hepatocarcinogenesis, prevention of HBV infection is a key step to reduce the incidence of liver cancer. There is a renewed interest regarding the understanding of various steps of the HBV replication cycle, as well as specific virus-host cell interactions, to define new targets and develop new antiviral drugs. Basically, the HBV covalently closed circular DNA (HBV cccDNA) is pivotal for persistent HBV infection and recurrence by the end of treatment. As far as we know, the cccDNA usually organizes into a minichromosome with histone 3 and $\mathrm{H} 4$ proteins and other nonhistone proteins, such as $\mathrm{HBx}, \mathrm{HBV}$ core protein, and host transcription factors ${ }^{[57]}$. Even though recent therapies can successfully control the viral replication, but they fail to eliminate cccDNA completely. Demonstrating the molecular mechanisms and screening critical factors involved in cccDNA may make it come true to develop more precisely targeted therapeutic strategies and cure HBV-related HCC patients ${ }^{[58]}$. It covers a series of inhibition of viral replication processes such as entry inhibitors, capsid assembly modulators, approaches aiming at the secretion of viral envelope proteins, drugs targeting HBV cccDNA, and siRNAs targeting viral transcripts. Restoration of immune responses is a complementary approach. HBV chronic infection and high viral load have been associated with higher levels of soluble programmed cell death protein 1, and this results 
in cytotoxic T-cell inhibition and 6.3-fold increase in risk for HCC development ${ }^{[59]}$. Using HBV infection models in vitro and in vivo, new targets and compounds will be available ${ }^{[60]}$.

HBx plays a crucial role in the various signal transduction pathways and HBV-induced hepatocarcinogenesis ${ }^{[61]}$. HBx accelerates the development of hepatoma ${ }^{[62]}$. HBx-elevated male-specific lethal 2 can strengthen HBV replication by regulating cccDNA in liver cancer cells, resulting in the development of $\mathrm{HCC}^{[63]}$. Moreover, we report that anti-HBx in sera may serve as one of the markers involving HBV-related liver cirrhosis and liver cancer ${ }^{[64]}$. Developing drugs targeting HBx is crucial for HBV-related HCC therapy. Two types of drugs, conventional interferon, and nucleoside analogs, have become available for the treatment of chronic hepatitis $\mathrm{B}$ infection. We also report that anti-HBV drugs such as entecavir, telbivudine and IFN- $\alpha 2 b$ inhibit the tumor growth of HBV-related HCC through depressing $\mathrm{HBx}^{[65]}$. The finding gives innovative insights into the mechanisms of anti-HBV drugs in HCC therapy.

\section{Molecular targets for the immunotherapy}

The research about a cohort of 956 HCC patients, 25\% had high expression of programmed deathligand-1 (PD-L1) and programmed cell death protein-1 (PD-1) in HCC tissues. Moreover, the study found that infiltrating $\mathrm{CD}^{+}$TILs (tumor-infiltrating lymphocyte) could induce PD-L1 expression via IFN- $\gamma^{[6]}$. Icotinib decreases the growth of hepatoma cells in vitro and in vivo, relying on EGFR activation and PDL1 expression ${ }^{[67]}$. Thus, the PD-1/PD-L1 pathway is available for prognosis and therapy in HCC. Patients with positive PD-L1 expression had significantly poorer DFS and overall survival (OS) than PD-L1 negative patients. The median DFS and OS were 14.9 and 29.6 months for PD-L1 positive patients compared with not reached and 59.4 months for PD-L1 negative patients, thus confirming the findings of the prognostic value of PD-1/PDL-1 in $\mathrm{HCC}^{[68]}$. Currently, nivolumab, a monoclonal antibody targeting PD-1, obtained an accelerated FDA approval in view of tumor response and durability for the therapy of HCC patients already treated with sorafenib in the phase $1 / 2$ single-arm CheckMate 040 study ${ }^{[69]}$. Nivolumab and pembrolizumab targeted PD-1 are ongoing in clinic ${ }^{[70]}$.

\section{Clinical trial status of molecular-targeted agents}

Tivantinib as the first drug was used to a phase III trial grounded in receptor overexpression analyses after disease progression on sorafenib in $\mathrm{HCC}^{[71]}$. Lenvatinib as an oral multikinase inhibitor for differentiated thyroid cancer and renal cell cancer treatment initially was approved. In a phase 2 trial of HCC patients in Japan and South Korea, lenvatinib treatment was obtained with a $37 \%$ response rate (by mRECIST), a median TTP of 7.4 months, and an available toxicity profile ${ }^{[72]}$. In addition, Regorafenib as the first agent showed a good survival benefit over placebo in patients progressing on sorafenib ${ }^{[73]}$. Regorafenib acting as an oral multikinase inhibitor, largely interdicted the activity of multiple protein kinases including tumor proliferation, metastasis, angiogenesis, microenvironment, and tumor immunity. Regorafenib exhibited a favorable survival regardless of the last dose of prior sorafenib (HR 0.67 for $800 \mathrm{mg} / \mathrm{day}$; 0.68 for $<800 \mathrm{mg} /$ day) ${ }^{[74]}$. Further approvals are coming, with good results from phase 3 trials evaluating cabozantinib and ramucirumab in the second-line setting. Cabozantinib, a small-molecule multikinase inhibitor was better than the placebo in the randomized phase 3 CELESTIAL trial ${ }^{[75]}$. Based on the trial analyses 707 advanced HCC patients previously received sorafenib treatment, cabozantinib distinctly increased OS over placebo (10.2 months vs. 8.0 months, respectively, $P=0.0049)^{[76]}$, as shown in Table 2.

\section{Sorafenib}

Sorafenib as a molecular-targeted agent can attenuate HCC proliferation and angiogenesis by inhibiting RAF serine threonine kinase and VEGF, PDGF, Flt-3, c-Kit receptor tyrosine kinase, getting approved in Europe and North America in 2007 and in Japan on May 20, 2009. To our delight, a subanalysis of the SHARP study, such as sorafenib in combination with resection, ablation, transcatheter arterial chemoembolization or hepatic arterial infusion chemotherapy, will overtly extend the overall survival in early-, intermediate- or 
Table 2. Overview of clinical trial agents for hepatocellular carcinoma therapy

\begin{tabular}{|c|c|c|c|}
\hline Agents & $\begin{array}{c}\text { Developmental } \\
\text { status }\end{array}$ & Targets & Outcomes \\
\hline Nivolumab & Phase III & $\mathrm{PD}-1^{[69]}$ & Ongoing $^{[70]}$ \\
\hline Pembrolizumab & Phase III & $P D-1^{[76]}$ & Ongoing ${ }^{[70]}$ \\
\hline Ramucirumab & Phase III & VEGFR2 $2^{[77]}$ & Survival benefit for the subgroup with AFP $\geq 400 \mathrm{ng} / \mathrm{mL}^{[79]}$ \\
\hline Lenvatinib & Phase III & VEGFR1-3, FGFR1-4, PDGFR $\alpha$, RET and KIT ${ }^{[70]}$ & Non-inferior OS, improved PFS, TTP, and ORR ${ }^{[70]}$ \\
\hline Cabozantinib & Phase III & TIE-1, TIE-2, FLT3, c-MET, KIT, RET and VEGFR ${ }^{[78]}$ & Significant improvement in OS, PFS, and ORR ${ }^{[75]}$ \\
\hline Tivantinib & Phase III & $\mathrm{c}-\mathrm{MET}^{[71]}$ & Improved OS and PFS ${ }^{[71]}$ \\
\hline
\end{tabular}

HCC: hepatocellular carcinoma; OS: overall survival; ORR: objective response rate; PFS: progression-free survival; TKI: tyrosine kinase inhibitor; TTP: time to progression

advanced-stage $\mathrm{HCCs}^{[80]}$. However, it increases the potential risk of invasion and metastasis of HCCalthough it significantly delays tumor progression time ${ }^{[81]}$.

\section{FUTURE CHALLENGES}

Although the effective diagnosis and therapies have been developed in HCC at present, it is unsatisfied to improve the patients' survival. The challenges in the field of diagnosis and therapy for HCC are still ongoing. Therefore, developing new diagnostic approach and drugs are urgent. About gene diagnosis of HCC, highthroughput means combined with bioinformatics methods will be used to find out the root cause of HCC in large-scale sample research. Clinically, it is necessary to monitor the biomarkers in the development of HCC involving treatment and prognosis, but not only in the early stage. It is vital to develop kits for determining the replication activity of HBV (or HCV) and HBV cccDNA to evaluate the risk of HCC incidence. For HCC therapy, identifying innovative targets and combination with multiple drugs are still needed in the treatment strategy. Effective combination of antiviral therapies with anti-inflammation drugs involving inflammation factors is available to treat chronic HBV-related HCC. It is necessary to examine the sensitivity, specificity, predictive value positive, predictive value negative, and validity of any candidate biomarker in a large pool of HCC patients with or without HBV infection, furthermore, it is also important to follow up large patients with HBV or other risk factor exposure for the prediction of occurrence and postoperative recurrence of HCC using representative markers. If biomarkers are valid, it is necessary to develop kits for molecular diagnosis, monitoring the efficacy, prognosis and treatments of HCC patients.

\section{CONCLUSION}

In summary, this review lists the recent progresses in gene diagnosis and therapy for HCC. The achievements include the recent novel biomarkers and novel therapeutic strategies for HCC, such as AFP, AFP-L3, GPC3, HSP90, DKK1, PON1, etc. Moreover, epigenetic regulation, signal pathway, cellular and molecular targets for the immunotherapy, tumor microenviroment and genome sequencing analysis may also serve as the molecular expression signatures in clinical practice. More studies are necessary to find new biomarkers for prognosis and treatment response in patients under standard treatment of sorafenib. The new clinical trials for other molecular-targeted agents, including pembrolizumab, nivolumab, tivantinib, lenvatinib, cabozantinib, and ramucirumab, are ongoing in clinic. Anti-HBV drugs are available in the therapy of HBVrelated HCC.

\section{DECLARATIONS}

\section{Authors' contributions}

Drafted the outline of this review: Zhang XD

Drafted the manuscript: Zhang XD, Zhao M

Finalized the manuscript: Zhang XD, Zhao M 


\section{Availability of data and materials}

Not applicable.

\section{Financial support and sponsorship}

None.

\section{Conflicts of interest}

All authors declare that there are no conflicts of interest.

\section{Ethical approval and consent to participate}

Not applicable.

\section{Consent for publication}

Not applicable.

\section{Copyright}

(c) The Author(s) 2019.

\section{REFERENCES}

1. Petrick JL, Kelly SP, Altekruse SF, McGlynn KA, Rosenberg PS. Future of hepatocellular carcinoma incidence in the United States forecast through 2030. J Clin Oncol 2016;34:1787-94.

2. Xu Y, Bu X, Dai C, Shang C. High serum microRNA-122 level is independently associated with higher overall survival rate in hepatocellular carcinoma patients. Tumor Biol 2015;36:4773-6.

3. Hamid AS, Tesfamariam IG, Zhang Y, Zhang ZG. Aflatoxin B1-induced hepatocellular carcinoma in developing countries: geographical distribution, mechanism of action and prevention. Oncol Lett 2013;5:1087-92.

4. Mak LY, Cruz-Ramon V, Chinchilla-Lopez P, Torres HA, LoConte NK, et al. Global epidemiology, prevention, and management of hepatocellular carcinoma. Am Soc Clin Oncol Educ Book 2018;38:262-79.

5. Crocetti L, Bargellini I, Cioni R. Loco-regional treatment of HCC: current status. Clin Radiol 2017;72:626-35.

6. Juarez-Hernandez E, Motola-Kuba D, Chavez-Tapia NC, Uribe M, Barbero Becerra V. Biomarkers in hepatocellular carcinoma: an overview. Expert Rev Gastroenterol Hepatol 2017;11:549-58.

7. Borel F, Konstantinova P, Jansen PL. Diagnostic and therapeutic potential of miRNA signatures in patients with hepatocellular carcinoma. J Hepatol 2012;56:1371-83.

8. Manini MA, Sangiovanni A, Fornari F, Piscaglia F, Biolato M, et al. Clinical and economical impact of 2010 AASLD guidelines for the diagnosis of hepatocellular carcinoma. J Hepatol 2014;60:995-1001.

9. Mitchell PS, Parkin RK, Kroh EM, Fritz BR, Wyman SK, et al. Circulating microRNAs as stable blood-based markers for cancer detection. Proc Nati Acadof Sci U S A 2008;105:10513-8.

10. Bianchi F, Nicassio F, Marzi M, Belloni E, Dall'olio V, et al. A serum circulating miRNA diagnostic test to identify asymptomatic high-risk individuals with early stage lung cancer. EMBO Mol Med 2011;3:495-503.

11. Ji J, Zhao L, Budhu A, Forgues M, Jia HL, et al. Let-7g targets collagen type I alpha2 and inhibits cell migration in hepatocellular carcinoma. J Hepatol 2010;52:690-7.

12. Zhang J, Yang Y, Yang T, Liu Y, Li A, et al. microRNA-22, downregulated in hepatocellular carcinoma and correlated with prognosis, suppresses cell proliferation and tumourigenicity. Br J Cancer 2010;103:1215-20.

13. Ji J, Shi J, Budhu A, Yu Z, Forgues M, et al. MicroRNA expression, survival, and response to interferon in liver cancer. N Engl J Med 2009;361:1437-47.

14. Xiong Y, Fang JH, Yun JP, Yang J, Zhang Y, et al. Effects of microRNA-29 on apoptosis, tumorigenicity, and prognosis of hepatocellular carcinoma. Hepatology 2010;51:836-45.

15. Li D, Liu X, Lin L, Hou J, Li N, et al. MicroRNA-99a inhibits hepatocellular carcinoma growth and correlates with prognosis of patients with hepatocellular carcinoma. J Biol Chem 2011;286:36677-85.

16. Zheng F, Liao YJ, Cai MY, Liu YH, Liu TH, et al. The putative tumour suppressor microRNA-124 modulates hepatocellular carcinoma cell aggressiveness by repressing ROCK2 and EZH2. Gut 2012;61:278-89.

17. Wong CC, Wong CM, Tung EK, Au SL, Lee JM, et al. The microRNA miR-139 suppresses metastasis and progression of hepatocellular carcinoma by down-regulating Rho-kinase 2. Gastroenterology 2011;140:322-31.

18. Jia YS, Liu HL, Zhuang QS, Xu SQ, Yang ZH, et al. Tumorigenicity of cancer stem-like cells derived from hepatocarcinoma is regulated by microRNA-145. Oncol Rep 2012;27:1865-72.

19. Wang C, Song B, Song W, Liu J, Sun A, et al. Underexpressed microRNA-199b-5p targets hypoxia-inducible factor-1alpha in hepatocellular carcinoma and predicts prognosis of hepatocellular carcinoma patients. J Gastroenterol Hepatol 2011;26:1630-7.

20. Li QJ, Zhou L, Yang F, Wang GX, Zheng H, et al. MicroRNA-10b promotes migration and invasion through CADM1 in human hepatocellular carcinoma cells. Tumor Biol 2012;33:1455-65. 
21. Chen L, Jiang M, Yuan W, Tang H. miR-17-5p as a novel prognostic marker for hepatocellular carcinoma. J Invest Surg 2012;25:156-61.

22. Karakatsanis A, Papaconstantinou I, Gazouli M, Lyberopoulou A, Polymeneas G, et al. Expression of microRNAs, miR-21, miR-31, miR-122, miR-145, miR-146a, miR-200c, miR-221, miR-222, and miR-223 in patients with hepatocellular carcinoma or intrahepatic cholangiocarcinoma and its prognostic significance. Mole Carcinog 2013;52:297-303.

23. Liu S, Guo W, Shi J, Li N, Yu X, et al. MicroRNA-135a contributes to the development of portal vein tumor thrombus by promoting metastasis in hepatocellular carcinoma. J Hepatol 2012;56:389-96.

24. Han ZB, Chen HY, Fan JW, Wu JY, Tang HM, et al. Up-regulation of microRNA-155 promotes cancer cell invasion and predicts poor survival of hepatocellular carcinoma following liver transplantation. J Cancer Res Clin Oncol 2012;138:153-61.

25. Wang J, Li J, Shen J, Wang C, Yang L, et al. MicroRNA-182 downregulates metastasis suppressor 1 and contributes to metastasis of hepatocellular carcinoma. BMC Cancer 2012;12:227.

26. Wong QWL, Ching AK, Chan AW, Choy KW, To KF, et al. MiR-222 overexpression confers cell migratory advantages in hepatocellular carcinoma through enhancing AKT signaling. Clin Cancer Res 2010;16:867-75.

27. Toffanin S, Hoshida Y, Lachenmayer A, Villanueva A, Cabellos L, et al. MicroRNA-based classification of hepatocellular carcinoma and oncogenic role of miR-517a. Gastroenterology 2011;140:1618-28.

28. Xie Y, Yao Q, Butt AM, Guo J, Tian Z, et al. Expression profiling of serum microRNA-101 in HBV-associated chronic hepatitis, liver cirrhosis, and hepatocellular carcinoma. Cancer Biol Ther 2014;15:1248-55.

29. Li L, Guo Z, Wang J, Mao Y, Gao Q. Serum miR-18a: a potential marker for hepatitis B virus-related hepatocellular carcinoma screening. Dig Dis Sci 2012;57:2910-6.

30. Su ZX, Zhao J, Rong ZH, Geng WM, Wu YG, et al. Upregulation of microRNA-25 associates with prognosis in hepatocellular carcinoma. Diagn Pathol 2014;9:47.

31. Han ZB, Chen HY, Fan JW, Wu JY, Tang HM, et al. Up-regulation of microRNA-155 promotes cancer cell invasion and predicts poor survival of hepatocellular carcinoma following liver transplantation. J Cancer Res Clin Oncol 2012;138:153-61.

32. Yamamoto Y, Kosaka N, Tanaka M, Koizumi F, Kanai Y, et al. MicroRNA-500 as a potential diagnostic marker for hepatocellular carcinoma. Biomarkers 2009;14:529-38.

33. Li LM, Hu ZB, Zhou ZX, Chen X, Liu FY, et al. Serum microRNA profiles serve as novel biomarkers for HBV infection and diagnosis of HBV-positive hepatocarcinoma. Cancer Res 2010;70:9798-807.

34. Tsai MC, Manor O, Wan Y, Mosammaparast N, Wang JK, et al. Long noncoding RNA as modular scaffold of histone modification complexes. Science 2010;329:689-93.

35. Rinn JL, Kertesz M, Wang JK, Squazzo SL, Xu X, et al. Functional demarcation of active and silent chromatin domains in human HOX loci by noncoding RNAs. Cell 2007;129:1311-23.

36. Li Y, Wang Z, Shi H, Li H, Li L, et al. HBXIP and LSD1 scaffolded by lncRNA Hotair mediate transcriptional activation by c-Myc. Cancer Res 2016;76:293-304.

37. Yang Z, Zhou L, Wu LM, Lai MC, Xie HY, et al. Overexpression of long non-coding RNA Hotair predicts tumor recurrence in hepatocellular carcinoma patients following liver transplantation. Ann Surg Oncol 2011;18:1243-50.

38. Xie H, Ma HW, Zhou DQ. Plasma HULC as a promising novel biomarker for the detection of hepatocellular carcinoma. Biomed Res Int 2013;2013:136106.

39. Cui M, Xiao Z, Wang Y, Zheng M, Song T, et al. Long noncoding RNA HULC modulates abnormal lipid metabolism in hepatoma cells through an miR-9-mediated RXRA signaling pathway. Cancer Res 2015;75:846-57.

40. Du Y, Kong G, You X, Zhang S, Zhang T, et al. Elevation of highly up-regulated in liver cancer (HULC) by hepatitis B virus X protein promotes hepatoma cell proliferation via down-regulating p18. J Biol Chem 2012;287:26302-11.

41. Wang Y, Chen F, Zhao M, Yang Z, Li J, et al. The long noncoding RNA HULC promotes liver cancer by increasing the expression of the HMGA2 oncogene via sequestration of the microRNA-186. J Biol Chem 2017;292:15395-407.

42 Zhao J, Greene CM, Gray SG, Lawless MW. Long noncoding RNAs in liver cancer: what we know in 2014. Expert Opin Ther Targets 2014; 18:1207-18.

43. Hernandez-Vargas H, Lambert MP, Le Calvez-Kelm F, Gouysse G, McKay-Chopin S, et al. Hepatocellular carcinoma displays distinct DNA methylation signatures with potential as clinical predictors. PLoS One 2010;5:e9749.

44. Nagashio R, Arai E, Ojima H, Kosuge T, Kondo Y, et al. Carcinogenetic risk estimation based on quantification of DNA methylation levels in liver tissue at the precancerous stage. Int J Cancer 2011;129:1170-9.

45. Shen J, Wang S, Zhang YJ, Kappil M, Wu HC, et al. Genome-wide DNA methylation profiles in hepatocellular carcinoma. Hepatology 2012;55:1799-808.

46. He C, Xu J, Zhang J, Xie D, Ye H, et al. High expression of trimethylated histone H3 lysine 4 is associated with poor prognosis in hepatocellular carcinoma. Hum Pathol 2012;43:1425-35.

47. Cai MY, Hou JH, Rao HL, Luo RZ, Li M, et al. High expression of H3K27me3 in human hepatocellular carcinomas correlates closely with vascular invasion and predicts worse prognosis in patients. Mol Med 2011;17:12-20.

48. Zhang ZQ, Meng H, Wang N, Liang LN, Liu LN, et al. Serum microRNA 143 and microRNA 215 as potential biomarkers for the diagnosis of chronic hepatitis and hepatocellular carcinoma. Diagn Pathol 2014;9:1-7.

49. Taketa K, Endo Y, Sekiya C, Tanikawa K, Koji T, et al. A collaborative study for the evaluation of lectin-reactive alpha-fetoproteins in early detection of hepatocellular carcinoma. Cancer Res 1993;53:5419-23.

50. Caviglia GP, Abate ML, Petrini E, Gaia S, Rizzetto M, et al. Highly sensitive alpha-fetoprotein, Lens culinaris agglutinin-reactive fraction of alpha-fetoprotein and des-gamma-carboxyprothrombin for hepatocellular carcinoma detection. Hepatol Res 2016;46:E130-5.

51. Capurro M, Wanless IR, Sherman M, Deboer G, Shi W, et al. Glypican-3: a novel serum and histochemical marker for hepatocellular carcinoma. Gastroenterology 2003;125:89-97.

52. Hippo Y, Watanabe K, Watanabe A, Midorikawa Y, Yamamoto S, et al. Identification of soluble NH2-terminal fragment of glypican-3 as a serological marker for early-stage hepatocellular carcinoma. Cancer Res 2004;64:2418-23. 
53. Fu Y, Xu X, Huang D, Cui D, Liu L, et al. Plasma heat shock protein 90alpha as a biomarker for the diagnosis of liver cancer: an official, large-scale, and multicenter clinical trial. EBioMedicine 2017;24:56-63.

54. Shen Q, Fan J, Yang XR, Tan Y, Zhao W, et al. Serum DKK1 as a protein biomarker for the diagnosis of hepatocellular carcinoma: a large-scale, multicentre study. Lancet Oncol 2012;13:817-26.

55. Abdel Wahab AHA, El-Halawany MS, Emam AA, Elfiky A, Abd Elmageed ZY. Identification of circulating protein biomarkers in patients with hepatocellular carcinoma concomitantly infected with chronic hepatitis C virus. Biomarkers 2017;22:621-8.

56. Zhang S, Jiang K, Zhang Q, Guo K, Liu Y. Serum fucosylated paraoxonase 1 as a potential glycobiomarker for clinical diagnosis of early hepatocellular carcinoma using ELISA Index. Glycoconj J 2015;32:119-25.

57. Zhang W, Chen J, Wu M, Zhang X, Zhang M, et al. PRMT5 restricts hepatitis B virus replication through epigenetic repression of covalently closed circular DNA transcription and interference with pregenomic RNA encapsidation. Hepatology 2017;66:398-415.

58. Ganem D, Prince AM. Hepatitis B virus infection - natural history and clinical consequences. N Engl J Med 2004;351:351.

59. Cheng HY, Kang PJ, Chuang YH, Wang YH, Jan MC, et al. Circulating programmed death-1 as a marker for sustained high hepatitis B viral load and risk of hepatocellular carcinoma. PLos One 2014;9:e95870.

60. Durantel D, Zoulim F. New antiviral targets for innovative treatment concepts for hepatitis B virus and hepatitis delta virus. J Hepatol 2016;64:S117-S131.

61. Zhang X, Zhang H, Ye L. Effects of hepatitis B virus X protein on the development of liver cancer. J Lab Clin Med 2006;147:58-66.

62. Zhang XD, Wang Y, Ye LH. Hepatitis B virus X protein accelerates the development of hepatoma. Cancer Biol Med 2014;11:182-90.

63. Gao Y, Feng J, Yang G, Zhang S, Liu Y, et al. Hepatitis B virus X protein-elevated MSL2 modulates hepatitis B virus covalently closed circular DNA by inducing degradation of APOBEC3B to enhance hepatocarcinogenesis. Hepatology 2017;66:1413-29.

64. Zhang H, Wu LY, Zhang S, Qiu LY, Li N, et al. Anti-Hepatitis B virus X protein in sera is one of the markers of development of liver cirrhosis and liver cancer mediated by HBV. J Biomed Biotechnol 2009;2009:289068.

65. Zhang S, Gao S, Zhao M, Liu Y, Bu Y, et al. Anti-HBV drugs suppress the growth of HBV-related hepatoma cells via down-regulation of hepatitis B virus X protein. Cancer Lett 2017;392:94-104.

66. Xie QK, Zhao YJ, Pan T, Lyu N, Mu LW, et al. Programmed death ligand 1 as an indicator of pre-existing adaptive immune responses in human hepatocellular carcinoma. Oncoimmunology 2016;5:e1181252.

67. Sun J, Jiang W, Tian D, Guo Q, Shen Z. Icotinib inhibits the proliferation of hepatocellular carcinoma cells in vitro and in vivo dependently on EGFR activation and PDL1 expression. Onco Targets Ther 2018;11:8227-37.

68. Mocan T, Sparchez Z, Craciun R, Bora CN, Leucuta DC. Programmed cell death protein-1 (PD-1)/programmed death-ligand-1 (PDL1) axis in hepatocellular carcinoma: prognostic and therapeutic perspectives. Clin Transl Oncol 2018. [DOI: 10.1007/s12094-0181975-4]

69. El-Khoueiry AB, Sangro B, Yau T, Crocenzi TS, Kudo M, et al. Nivolumab in patients with advanced hepatocellular carcinoma (CheckMate 040): an open-label, non-comparative, phase 1/2 dose escalation and expansion trial. Lancet 2017;389:2492-502.

70. Raoul JL, Kudo M, Finn RS, Edeline J, Reig M, et al. Systemic therapy for intermediate and advanced hepatocellular carcinoma: sorafenib and beyond. Cancer Treat Rev 2018;68:16-24.

71. Best J, Schotten C, Lohmann G, Gerken G, Dechene A. Tivantinib for the treatment of hepatocellular carcinoma. Expert Opin Pharmacother 2017;18:727-33.

72. Ikeda K, Kudo M, Kawazoe S, Osaki Y, Ikeda M, et al. Phase 2 study of lenvatinib in patients with advanced hepatocellular carcinoma. Journal of Gastroenterology 2017;52:512-9.

73. Bruix J, Qin S, Merle P, Granito A, Huang YH, et al. Regorafenib for patients with hepatocellular carcinoma who progressed on sorafenib treatment (RESORCE): a randomised, double-blind, placebo-controlled, phase 3 trial. Lancet 2017;389:56-66.

74. Finn RS, Merle P, Granito A, Huang YH, Bodoky G, et al. Outcomes of sequential treatment with sorafenib followed by regorafenib for HCC: Additional analyses from the phase III RESORCE trial. J Hepatol 2018;69:353-8.

75. Abou-Alfa GK, Meyer T, Cheng AL, El-Khoueiry A, Rimassa L, et al. Cabozantinib (C) versus placebo (P) in patients (pts) with advanced hepatocellular carcinoma (HCC) who have received prior sorafenib: Results from the randomized phase 3 CELESTIAL trial. J Clin Oncol 2018;36.

76. Khoja L, Butler MO, Kang SP, Ebbinghaus S, Joshua AM. Pembrolizumab. J Immunother Cancer 2015;3:36.

77. Zhu AX, Ryoo BY, Yen CJ, Kudo M, Poon RTP, et al. Ramucirumab (RAM) as second-line treatment in patients (pts) with advanced hepatocellular carcinoma (HCC): Analysis of patients with elevated alpha-fetoprotein (AFP) from the randomized phase III REACH study. J Clin Oncol 2015;33.

78. Yakes FM, Chen J, Tan J, Yamaguchi K, Shi Y, et al. Cabozantinib (XL184), a novel MET and VEGFR2 inhibitor, simultaneously suppresses metastasis, angiogenesis, and tumor growth. Mol Cancer Ther 2011;10:2298-308.

79. Zhu AX, Park JO, Ryoo BY, Yen CJ, Poon R, et al. Ramucirumab versus placebo as second-line treatment in patients with advanced hepatocellular carcinoma following first-line therapy with sorafenib (REACH): a randomised, double-blind, multicentre, phase 3 trial. Lancet Oncol 2015; 16:859-70.

80. Kudo M, Ueshima K. Positioning of a molecular-targeted agent, sorafenib, in the treatment algorithm for hepatocellular carcinoma and implication of many complete remission cases in Japan. Oncology 2010;78 Suppl 1:154-66.

81. Lu L, Lu M, Pei Y, Chen J, Qin L, Zhu W, Jia H. Down-regulation of SDF1- $\alpha$ expression in tumor microenvironment is associated with aspirin-mediated suppression of the pro-metastasis effect of sorafenib in hepatocellular carcinoma. Acta Biochim Biophys Sin 2015;47:988-96 